\title{
Clinical Guidelines Written by Residents
}

David W. Andrews MD

Thomas Jefferson University

Follow this and additional works at: https://jdc.jefferson.edu/jhnj

Part of the Neurology Commons

Let us know how access to this document benefits you

\section{Recommended Citation}

Andrews MD, David W. (2008) "Clinical Guidelines Written by Residents," JHN Journal: Vol. 4 : Iss. 2 , Article 7.

DOI: https://doi.org/10.29046/JHNJ.004.2.003

Available at: https://jdc.jefferson.edu/jhnj/vol4/iss2/7

This Article is brought to you for free and open access by the Jefferson Digital Commons. The Jefferson Digital Commons is a service of Thomas Jefferson University's Center for Teaching and Learning (CTL). The Commons is a showcase for Jefferson books and journals, peer-reviewed scholarly publications, unique historical collections from the University archives, and teaching tools. The Jefferson Digital Commons allows researchers and interested readers anywhere in the world to learn about and keep up to date with Jefferson scholarship. This article has been accepted for inclusion in JHN Journal by an authorized administrator of the Jefferson Digital Commons. For more information, please contact: JeffersonDigitalCommons@jefferson.edu. 


\title{
Clinical Guidelines Written by Residents
}

\author{
David W. Andrews, MD \\ Department of Neurological Surgery, Thomas Jefferson University, Philadelphia, Pennsylvania
}

\begin{abstract}
"Variation" is an innocent word that that can represent many levels of frustration to the clinician. Variation among patients is the least of these; the physician expects patients and their individual problems to be as diverse as the human race itself. Variation within a practice should be due to matching the specific needs of the specific patient. Other variations can mean trouble if they represent differences in understanding of the problem among clinicians and other allied health practitioners. These differences could be between institutions or even between shifts within one institution.

The Tufts Health Care Institute (THCI) is a non-profit organization which provides educational tools for medical and surgical residents across the US. One of these tools teaches clinical practice guidelines, defined by the Institute of Medicine as "systematically developed statements to assist practitioner and patient decisions about appropriate health care for specific clinical circumstances." . One of the authors of the Clinical Guidelines module is David B. Nash, Professor of Health Policy here at Thomas Jefferson University.

The Jefferson Department of Neurological Surgery has been participating in the THCI program for the past few years, mentored by Dr. David W. Andrews of the department. Because surgery and medicine have different emphases, Dr. Andrews has found that some of the THCI modules are more appropriate than others for the neurosurgical residents, and that the Clinical Guidelines module in particular has generated both enthusiasm and good results. A selection of the TJU neurosurgical resident-generated guidelines are presented here. These guidelines have not officially been adopted by the department or by any institution that we know of, but show how clinical problems can be identified and solved.
\end{abstract}

\section{References}

1. (Institute of Medicine, 1990, p. 38) Institute of Medicine. Field MJ, Lohr KN (eds.) Clinical Practice Guidelines: Directions for a New Program, page 38. Committee to Advise the Public Health Service on

\section{Acknowledgement}

All graphics used are from commons.wikipedia.org under open licenses. The specific license of each graphic (GNU, Creative Commons) is available by looking up its URL.

\section{Anticonvulsant use in brain tumor patients}

Hugh Moulding, MD

More than 200,000 patients are diagnosed with primary or metastatic brain tumors each year in the United States. Of these patients, $20 \%$ to $40 \%$ will develop seizures at presentation, and another $20 \%$ to $40 \%$ will require treatment for seizures during their illness. Although the use of antiepileptic drugs (AEDs) in patients who have had seizures seems reasonable, the issue of prophylactic AED use for patients who have not had a seizure is an intensely debated subject.

At TJUH, we see the wide variety of practices in prophylactic use of anticonvulsants. Some physicians will use anticonvulsants and follow therapeutic levels, others will use a starting dose of AED and not follow levels, and still others will not use AED at all. The reason for the discrepancy is most likely multifactorial. There is little evidence to the benefit of AEDs in patients who have not had seizures when considering the side effect profile of AEDs. However, give the litigious climate of Philadelphia, many neurosurgeons are reluctant to leave a patient with an intracerebral lesion without antiepileptic coverage. Additionally, many neurosurgeons are creatures of habit, trained to practice a certain way, and have done so for many years during which "it has worked or them so far." A set of guidelines based on a literature search, where level I evidence is cited where possible, would help physicians to be more comfortable about changing their practice.

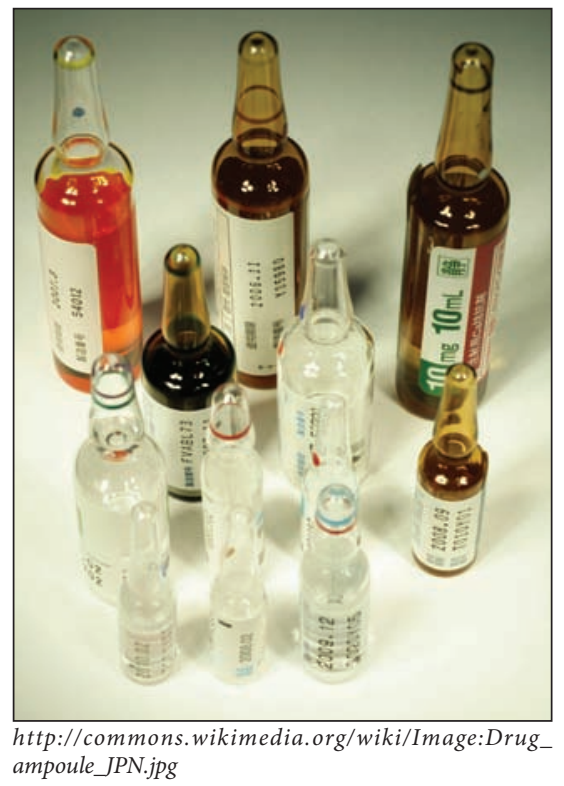


Since 2000, there have been practive guidelines in place approved by the American Academy of Neurology (see Ref 3) based on peer reviewed publications with level I evidence. Despite these practice parameters a recent survey reported that $81 \%$ of neurosurgeons and $53 \%$ of neurologists prescribed antiepileptic drugs (AEDs) prophylactically.

For the $20-40 \%$ of patients diagnosed with a brain tumor who have experienced a seizure, prophylactic use of AEDs is universally accepted. Seventy percent of brain tumor patients presenting with seizures will suffer recurrent seizures regardless of tumor type. However, patients that have not had a seizure still remain at risk and $20-45 \%$ will develop seizures. Some factors that have been shown to increase the likelihood of developing seizures are:

1. Tumor location, most likely in frontal, parietal or temporal lobes, especially parasagital meningiomas ( $74 \%$ seizure prevalence).

2. Number of tumors: increased number of tumors correlates to higher seizure risk.

3. Tumor histopathology: Slow growing tumors are at increased risk.

4. Age: younger patients more likely to seize.

One factor that is conspicuously absent from the list above is AED use. Of the twelve studies of Level I \& II data that examined this question, only one reported a significant difference in seizure frequency and this favored the nonAED group having a lower seizure risk. A meta-analysis performed on the four Level I studies showed an Odds Ratio of 1.09 with no statistical significance $(\mathrm{p}=0.9)$. Furthermore AED use is not benign; $23.8 \%$ of these patients experience side effects that warranted change or discontinuation of the AED. These side effects included rash (14\%), nausea and vomiting (5\%), encephalopathy (5\%), and myelosuppression (3\%).

\section{References \\ 1. Sperling MR, Ko J: Seizures and brain tumors. Seminars in Oncology. 33(3):333-41, 2006. \\ 2. Stevens GHJ: Antiepileptic therapy in patients with central nervous system malignancies. Current Neurology and Neuroscience Reports. 6(4):311-8, 2006. \\ 3. Glantz MJ, Cole BF, Forsyth PA, et al.: Practice parameter: anticonvulsant prophylaxis in patients with newly diagnosed brain tumors: report of the quality standards subcommittee of the American Academy of Neurology. Neurology. 54:1886-93, 2000}

\section{Cervical spondylotic myelopathy}

Amgad Hanna, MD

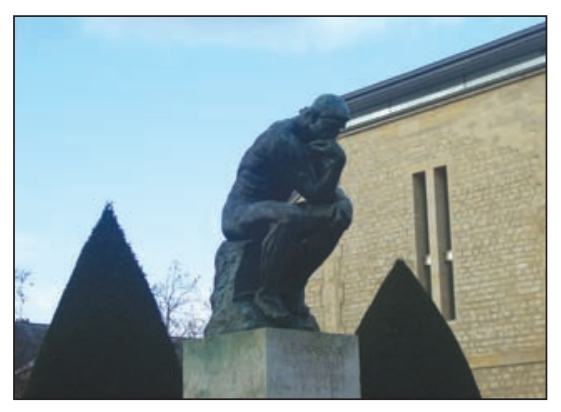

http://commons.wikimedia.org/wiki/Image:The Thinker_wideshot.jpg

Cervical spondylotic myelopathy is a common cause of major morbidity in middle-aged and elderly people. Various functional classifications are used to evaluate patients with cervical spondylosis. They are based on pure clinical data, and are used as a method of evaluation of the patient's progress during the course of the disease. None of these classifications defines criteria for the indication for surgery.

Patients with cervical myelopathy may be managed either conservatively or surgically. Conservative management includes medications (e..g, non-steroidal anti-inflammatories or corticosteroids), cervical orthotics, physical therapy, or injections. Surgery includes anterior and/or posterior decompression and/or fusion.

There remains considerable uncertainty regarding the decision to operate and the timing for surgery. This is left to the surgeon's discretion in most of the cases. Most surgeons would agree that patients with mild myelopathy should be managed conservatively and patients with severe myelopathy should be managed surgically. There is a large gray zone of moderate myelopathy where surgeons may decide about surgery or conservative management. The definition of mild, moderate, or severe myelopathy is vague in literature.

The timing of surgery is also controversial in literature. Advocates of early surgery try to stop disease progression, prevent further clinical deterioration of the patients, and prevent the risk of acute deterioration with neck injuries (central cord syndrome). Advocates of late surgery try to delay the potential complications of surgery like adjacent level disease, spinal cord injury, cerebrospinal fluid leak, infection, bleeding, and possible clinical deterioration.
We attempt to address the literature controversies regarding this topic, the severity of myelopathy, and the indications for surgery.

\section{Online search for resources}

1. National guideline clearinghouse. We searched for "cervical myelopathy". No guidelines were found for the management of cervical myelopathy. Related guidelines were found for cervical radiculopathy: Review criteria for cervical surgery for entrapment of a single nerve root. Olympia (WA): Washington State Department of Labor and Industries; 2004 June. 1p. The article states that "Cases of myelopathy should be referred for physician review".

2. The Cochrane Library. We searched for "cervical myelopathy". One article was found: Cochrane review on the role of surgery in cervical spondylotic radiculomyelopathy. Fouyas I, Statham P, Sandercock P. Spine 2002; 27 (7): 736-47

3. Medline search: www.ncbi.nlm.nih.gov. We searched for "management of cervical myelopathy"; 104 articles were found

\section{Summary of findings}

There are no current guidelines for the management of cervical spondylotic myelopathy. Several articles $(1,2)$ discussed the prognostic factors and presented surgical outcomes. These articles presented conflicting results as far as the prognostic significance of signal changes on MRI, age, and duration of symptoms.

Other studies discussed the indications and outcomes of different surgical approaches. Again, conflicting results were found. Fouyas et al. found no significant longterm differences between surgery and conservative management $^{3}$. Houten, Kim, Medow, Komotar, and Sekhon showed good results with surgical decompression ${ }^{2,4-7}$. However, there was no comparison to conservative management.

\section{Guidelines}

The two articles selected are references 2 and 3.

\section{Reference 2: Houten, Cooper (2003)}

Full reference: Laminectomy and posterior cervical plating for multilevel cervical spondylotic myelopathy and ossification of the posterior longitudinal ligament: effects on cervical alignment, spinal cord compression, and neurological outcome. Houten J, Cooper P. Neurosurgery 2003; 52 (5): 1081-8 


\section{Results}

- Multilevel cervical laminectomy and fusion for cervical spondylotic myelopathy provide minimal morbidity, excellent decompression, immediate stability, prevent kyphosis, and precludes further spondylosis.

\section{Strengths}

- Combined clinical and radiological criteria for outcome after surgery.

- Thirty months follow up.

\section{Weaknesses}

- Retrospective study.

- Small number of patients (38).

- No comparison group with conservative management.

- No consideration for bony fusion vs pseudoarthrosis.

\section{Reference 3: Fouyas, Statham, Sandercock (2002)}

Full reference: Cochrane review on the role of surgery in cervical spondylotic radiculomyelopathy. Fouyas I, Statham P, Sandercock P. Spine 2002; 27 (7): 736-47

\section{Final Guideline}

This guideline targets mainly neurosurgeons and orthopedic spine surgeons who need to decide for their patients whether to proceed with surgical or conservative management. It also targets primary care physicians to guide them as to which patients need to be referred for surgery.

\section{Evaluation of the severity of myelopathy:}

The following scoring system is proposed to evaluate the severity of cervical myelopathy:

\section{Comments}

- This is the first grading system to include both clinical and radiological data.

- Mild to moderate symptoms: mild pain or numbness. Severe symptoms: severe pain or numbness, loss of dexterity, difficulty in walking.

- Mild to moderate signs: hyper-reflexia, Hoffman's or Babinski's signs, mild weakness. Severe signs: significant weakness, spasticity.

- Moderate stenosis: Mid-sagittal diameter: 10-12 mm. Severe stenosis: Mid-sagittal diameter: $<10 \mathrm{~mm}$.

- The recommendations are based on the best available literature.

- This is just a guideline. Cases need to be managed individually, based on the whole

\section{Table 1. Score System}

\begin{tabular}{llllll}
\hline No Symptoms & 0 & Mild to Moderate Symptoms & 1 & Severe Symptoms & 2 \\
\hline No Signs & 0 & Mild to Moderate Signs & 1 & Severe Signs & 2 \\
\hline No stenosis & 0 & Moderate Stenosis & 1 & Severe Stenosis & 2 \\
\hline $\begin{array}{l}\text { No signal changes } \\
\text { on MRI }\end{array}$ & 0 & & Signal Changes on MRI & 2 \\
\hline
\end{tabular}

Total score 1-3: Conservative management. Total score 4-8: Surgery.

\section{Results}

- No significant difference in long term outcome between surgical and conservative management for cervical myelopathy.

\section{Strengths}

- Prospective randomized trials.

\section{Weaknesses}

- Patients represented in the studies had mainly mild functional deficit. They do not represent the population that usually benefits from surgery, who have moderate to severe myelopathy. clinical scenario, progression of the disease, and co-morbidities.

\section{References}

1. Effect of intramedullary signal changes on the surgical outcome of patients with cervical spondylotic myelopathy. Suri et al. Spine J 2003; 3 (1): 33-45

2. Laminectomy and posterior cervical plating for multileve cervical spondylotic myelopathy and ossification of the posterior longitudinal ligament: effects on cervical alignment, spinal cord compression, and neurological outcome. Houten J, Cooper P. Neurosurgery 2003; 52 (5): 1081-8

3. Cochrane review on the role of surgery in cervical spondylotic radiculomyelopathy. Fouyas I, Statham P, Sandercock P. Spine 2002; 27 (7): 736-47
4. Indications for circumferential surgery for cervical spondylotic myelopathy. Kim P, Alexander J. Spine J 2006; 6 Suppl: S 299-307

5. Surgical management of cervical myelopathy: indications and techniques for surgical corpectomy. Medow J, Trost G, Sandin J. Spine J 2006; 6 Suppl: S 233-41

6. Surgical management of cervical myelopathy: indications and techniques for laminectomy and fusion. Komotar R, Morcco J, Kaiser M. Spine J 2006; 6 Suppl: S 252-67

7. Posterior cervical decompression and fusion for circumferential spondylotic cervical stenosis: review of 50 consecutive cases. Sekhon L. J Clin Neurosci 2006; 13 (1): 23-30

\section{Does the treatment of carotid dissection require anticoagulation with coumadin or is antiplatelet therapy equally efficacious?}

\section{Aditya Pandey, MD}

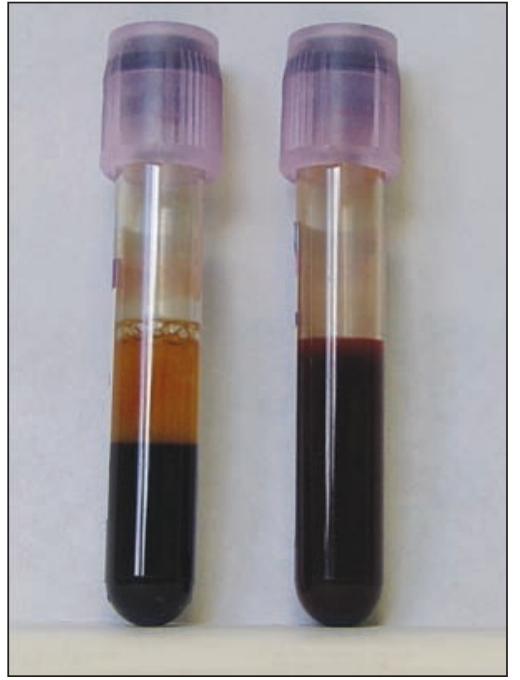

http://commons.wikimedia.org/wiki/Image:Blut-EDTA.jpg

Vessel dissection represents injury to the wall of the vessel either from the luminal side or adventitial (outside) side. Extracranial carotid and vertebral artery dissections represent the second leading cause of stroke in young adults. Its incidence is $2-3$ persons/ 100,000 / year. The mechanism of developing stroke within this patient population is from clot formation at the site of the injury to the vessel. The causes of carotid dissection are numerous, but it most commonly occurs due to a traumatic injury in an individual who has weakened vessels. To prevent such an event, patients are started on blood thinners: either antiplatelet (aspirin) or coumadin.

Depending on physician and surgeon preference, different anticoagulation therapy can be used for the same type of patient with carotid 
dissection. Those who use coumadin do so since they have had success in treating carotid dissection patients without stroke formation. The same is true of physicians utilizing antiplatelet therapy. The use of coumadin not only requires that patients have frequent blood tests to show level of blood thinning but also that the bleeding risks associated with it are higher. The goal of this analysis is to present the evidence for using coumadin versus aspirin in the treatment of carotid dissection.

\section{Benefits of Full Anticoagulation}

- anticoagulation leads to the prevention of stroke from the formation of clots at the site of the vessel injury

\section{Complications of Full Anticoagulation}

- anticoagulation could lead to further bleeding into the brain

\section{Specifics of Search}

- Search of National Clearinghouse Guidelines revealed one review but no specific guidelines

- Search of the Cochrane Database did not reveal any position statements on the subject

- The following key term searches on Pubmed led to numerous articles on the subject of treating carotid dissection with anticoagulation:

- "Extracranial carotid dissection"

- "Treatment of extracranial carotid dissection"

- "Anticoagulation for extracranial carotid dissection"

\section{Results}

1. Antithrombotic drugs for carotid artery dissection. Cochrane Database Syst Rev. 2000; (4): CD 000255

\section{Strengths}

- comprehensive review of case controlled studies and case series

- applies to carotid dissection patients who had undergone either anticoagulation with coumadin or antiplatelet therapy

Limitations

- no randomized control trials included in this study (No RCT have been performed addressing this issue)

\section{Summary}

- 26 eligible studies were reviewed (327 patients)
- No statistically significant difference in outcome (mortality/disability) between patients on antiplatelet therapy versus those on anticoagulation (coumadin)

- No difference in the occurrence of intracerebral hemorrhage between the two groups $(0 \%$ for antiplatelet group and $0.5 \%$ for anticoagulation group)

\section{Conclusion}

- no statistically significant difference in outcome or complication when comparing the anticoagulation versus the antiplatelet group of patients

2. Cervical Arterial Dissection: time for a therapeutic trial? Stroke. 2003 Dec; 34 (12): $2856-60$

\section{Strengths}

- prospective enrolled patients with carotid and vertebral dissections

- large number of patients $(n=116)$

- comparison of aspirin vs. anticoagulation

Weaknesses

- not a randomized control trial

- N might not be large enough to a small statistically significant difference

\section{Summary}

- Canadian Stroke Consortium prospectively enrolled patients with extra-cranial dissections and followed patients for one year. The endpoints being evaluated included: TIA, Stroke, or Death. 105 patients had complete follow up. In patients treated with anticoagulation the event rate was $8.3 \%$ versus those treated with aspirin where the event rate was $12.4 \%$. While there was an absolute difference of $4.1 \%$, this difference was not statistically significant.

\section{Conclusion}

There were no statistically significant differences between the outcome rates of patients on ASA versus those on anticoagulation.

1. Dissection of Cervical Arteries: Long-term follow-up study of 130 consecutive cases. Cerebrovasc Dis. 2006; 22 (2-3): 150-4

\section{Strengths}

- Prospective Study

- $\mathrm{N}=130$ cases

- Comparison of ASA vs. anticoagulation

Weaknesses

- not a randomized control trial

- F/U only 6 months

\section{Summary}

- 130 patients with angiographically proven cervical arterial dissection were followed for the events of stroke and death. There was no significant differences in outcome were found when comparing patients on aspirin versus the patients on anticoagulation.

\section{Conclusion}

In patients with proven carotid dissections, there is no difference in outcome between patients treated with ASA vs. those treated with anticoagulation.

1. Outcome of extracranial cervicocephalic arterial dissections: A follow-up study. Neurol Res. 2002 Jun; 24 (4): 395-8

\section{Strengths}

- comparison of ASA vs. anticoagulation

- long term f/u (nearly 10 years)

\section{Weaknesses}

- not a randomized control trial

- small patient population $(n=27)$

\section{Summary}

- 27 patients with extracrainal CAD who were treated and followed by the stroke service. Outcome was assessed using the modified Rankin Score and recurrent stroke and TIAs were also recorded. The outcome was favorable with either antiplatelet or anticoagulation.

\section{Conclusion}

Either antiplatelets or anticoagulation are equally effective in preventing strokes after carotid dissection,

\section{Final Guideline}

In individuals with a proven extracranial carotid dissection, aspirin therapy should be initiated instead of coumadin. The current literature on the subject shows no statistically significant difference in outcome of individuals treated with aspirin versus those treated with coumadin in the setting of carotid dissection. While the risks with coumadin are not higher, it still requires evaluation of the INR for accurate dosing. Such is not the requirement of antiplatelet therapy. In addition other medications (cimetidine) can affect the metabolism of coumadin thus leading to inappropriate levels of anticoagulation. Thus antiplatelet therapy is an effective method of preventing strokes in individuals with carotid dissection. 


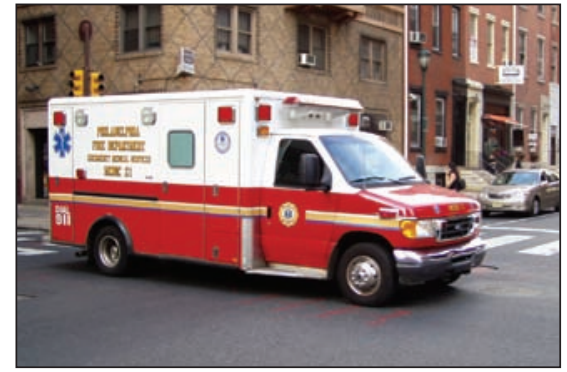

http://commons.wikimedia.org/wiki/Image:Philadelphia_Fire_ Department_medic_unit.JPG

\section{Would a standardized protocol for the inter-hospital transport of critically ill patients alter outcomes?}

Jack Klem, MD

The rapid evolution of healthcare technology has created a disparity among hospitals and thus led to the establishment of highly specialized quarternary institutions, termed "Centers of Excellence." This has created a need for the systematic triage and transport of certain critically ill patients that would not receive adequate care at a community hospital. Emergency Medical Services (EMS) has made the transport of patients a national issue due to regionalization, specialization, and facility designation by payers. Despite this, there exists a paucity of guidelines to direct the appropriate flow of patients to higher levels of care.

Diversity exists amongst transport teams and overall resource allocation. Interfacility transport is provided by a variety of levels and types of personnel and agencies. The medical condition of the patient is not always matched appropriately with the acuity of care provided by the transport team. Less severely ill patients are sometimes intubated and ventilated solely to facilitate ease and safety of transport.

In addition, the disease process of a particular diagnosis is not always understood prior to dispatching the appropriate means of transport (ie, air vs. ground). For example, patients with suspected aneurysmal subarachnoid hemorrhage require the most expeditious mode of transportation due the high risk of rerupture in the acute period. Moreover, certain diagnoses such as cerebellar hematoma with deteriorating neurological function require immediate transfer to the operating room with no need for an available ICU bed. Recognition of these "hyperacute" scenarios are critical to patient outcome.
Guidelines are necessary to standardize interfacility transport on at least the regional level. Several steps must be analyzed in order to establish a systems-based protocol for interfacility transfer. The type of transfer must be established (ie, hospital to hospital, rehab to hospital, clinic to hospital, etc.) Next, provider capabilities must match the patient's current and potential needs in order to provide safe and effective care during transport. This has been shown to impact outcomes in the transport of pediatric patients. On an administrative level, certification of necessity for transfer is a requirement for reimbursement by Medicare and Medicaid. This is directed by federal legislation outlined in the Consolidated Omnibus Budget Reconciliation Act (COBRA) and Emergency Medical Treatment and Labor Act (EMTALA). In summary, all of these factors must be integrated in order to develop a regional or even national plan for interfacility transport. This shall streamline resource allocation and potentially improve outcomes

\section{Online Search}

1. National Guidelines Clearinghouse was queried for "patient, hospital transport" and "interhospital transport"

2. Google was queried for "patient transport, guidelines" and "hospital transport"

\section{Study \#1: Warren at al (2004)}

Full reference: Warren et al. Guidelines for the inter- and intrahospital transport of critically ill patients. Crit Care Med 32:1 (2004) 256- 262.

\section{Purpose}

- Development of practice guidelines for inter- and intrahospital transport of the critically ill patient in order to establish an efficient, organized process supported by appropriate equipment and personnel. This would ultimately enhance patient safety.

Methods

- Synthesis of prospective clinical outcome studies, retrospective reviews, and anecdotal reports by a task force of experts providing consensus opinion.

\section{Results}

- A multidisciplinary team of physicians, nurses, respiratory therapists, hospital administration and the local emergency medical service must justify and coordinate the process by conducting a needs assessment of the referring hospital or clinic, asking whether transport is likely to alter the management or outcome of the patient. A patient must have a preliminary diagnosis that can be further refined and ultimately treated at a center that offers specialized diagnostic capability. Receiving hospitals should then proceed with a formalized plan addressing the following points:

pretransport coordination and communication

transport personnel

transport equipment:

monitoring during transport

documentation: The patient's medical record and relevant laboratory and radiographic studies must be copied for the accepting facility. It is also suggested to perform a COBRA/EMTALA checklist to ensure compliance with federal regulations.

- The above five points should be evaluated and refined regularly by the hospital using a standard quality improvement process. Many of the specific details from these guidelines are incorporated in the final guidelines below.

\section{Conclusions}

When services are required that exceed available resources at a particular hospital or clinic, a patient will be ideally transferred to a facility that has the necessary resources. The decision to transport a critically ill patient is based on the potential benefits (ie, higher level of technical/cognitive/procedural care) and weighed against the risks. Justification for transport must be established and several points must be addressed to ensure safe and efficient transfer.

\section{Strengths of study}

- Article provides clear, comprehensive guidelines discussed above for transporting patient within and between hospitals. Not only do these guidelines comply with federal regulations, but they also illustrate the importance of having an organized, efficient and standardized protocol that can be followed by any hospital.

Details of transport such as appropriate equipment and personnel are described in order to ensure utmost safety for the patient being transported. Again, this is an effort to standardize the entire process in order to eliminate "system-based" mistakes. For example, there exists much variability in the acuity of care provided 
by transport teams due to improper triage, insufficient equipment, and inexperienced personnel. To resolve this, this article takes the stance that patient transportation must be subjected to the same rigors as the aviation industry in eliminating systems errors by establishing a universal set of guidelines.

\section{Weaknesses of study}

- This is really the first effort in the literature to establish a universal set of guidelines and, as a result, no outcome studies are yet available.

- This article also assumes that every hospital has the same basic resources available to standardize patient transport. This becomes an issue with the transport of critically ill patients where the accepting hospital may have to provide the appropriately staffed and equipped transportation team.

\section{Study \#2: Fan et al (2006)}

Full reference: Fan et al. Outcomes of interfacility critical care adult patient transport: a systematic review. Critical Care 10 (2006) 1-7.

Purpose

- Determine the adverse events and important prognostic factors associated with interfacility transport of intubated and mechanically ventilated adult patients.

\section{Methods}

- A systematic review of multiple databases yielded 5 case-series comprising of a total of 245 patients. Two of the case-series were prospective in design.

\section{Results}

- Data was synthesized in a qualitative manner due to significant heterogeneity in study population, outcome events, and results. The most common indication for interfacility transport was a need for specialized investigations and interventions. Transport modalities included air (66\%), ground $(31 \%)$ and commercial aircraft (3\%). Transport teams included a physician in 3 of the 5 studies. Death during transport was rare $(n=1)$ and no other adverse events or significant therapeutic interventions were reported during transport. Of note, one study reported a 19\% (28/145) incidence of respiratory alkalosis upon arrival.

\section{Conclusion}

Insufficient data exists regarding the mortality, morbidity, and risk factors associated with the interfacility transport of critically ill patients. Further research is necessary to understand which patients are most at risk while being transported. Recognizing the types of events that can occur is an important step in patient preparation and planning.

\section{Strengths of study}

- First attempt to study outcomes of interhospital transport of critically ill patients

\section{Weaknesses of study}

- This is similar to a meta-analysis that combines different levels of evidence (ie, three retrospective studies plus two prospective studies) and attempts to draw meaningful conclusions. There must be one prospective trial that addresses the same patient population.

- This article looks only at adverse outcomes that occur while a patient is en route rather than considering the period just before and after transport. This under reports situations, for example, where some patients begin to deteriorate and are intubated immediately upon arrival.

\section{Study \#3: Ligtenberg et al (2005)}

Full reference: Ligtenberg et al. Quality of interhospital transport of critically ill patients: a prospective audit. Critical Care 9 (2005) 446-51.

\section{Purpose}

- Determine the adverse events and important prognostic factors associated with interfacility transport.

Methods

- prospective study describing 100 consecutive ICU transfers, of which $65 \%$ were mechanically ventilated and $38 \%$ on vasoactive drugs.

\section{Results}

- Data was collected on adverse events before, during, and after transport. 34\% of patients incurred adverse events with 6 deaths being reported within the first 24-hours after arrival. This study was different because not all patients were intubated and not all were accompanied by a physician as was the case in Fan et al. (2006).

\section{Conclusions}

Important factors for improvement were better overall communication between the referring/ receiving hospital and strict adherence to checklists/ published protocols.

\section{Strengths of study}

- Describes a single, prospective study involving the transfer of 100 patients into only one university center. This eliminates the inherent flaws of a combining several different studies.

- Information regarding adverse events was collected for 24-hours after arrival.

\section{Weaknesses of study}

- There was no stratification of diagnoses. For example, there is no discussion as to why each of the 34 adverse events occurred. For example, was this due to inadequate stabilization of the patient's medical condition prior to transport, due to inadequate care provided during transport, or merely patient disease?

Final Guidlines for inter-hospital transport of critically ill patients

Diagnosis

- Patient requires emergent surgical intervention that will occur immediately upon arrival to the accepting institution. This applies to diagnoses such as cerebellar hematoma, $\mathrm{EDH}$, or $\mathrm{SDH}$ in the setting of a rapidly deteriorating patient. This defines the highest acuity transfer wherein communication between physicians, OR staff, and transport personnel is critical to transporting a patient directly to the holding area and/or operating room in order to save the patient's life. Expending time to "make a bed" at the accepting institution will gravely impact the patient's outcome and thus must be overlooked in order to transport the patient as rapidly as possible to the operating suite.

- Patient requires emergent surgical intervention within 24-hours of arriving to the accepting institution. This applies to diagnoses such as SAH where cerebral angiogram with definitive treatment is performed within 24-hours of arrival. The caveat here is that these patients may develop hydrocephalus which sometimes cannot be treated at a referring institution and therefore requires emergent transport. Another diagnosis is cauda equina where a patient will require an MRI followed by surgery within 24-hours. 
Patient requires urgent imaging studies that may or may not require surgery in the setting of a neurologically and medically stable patient. This applies to diagnoses such as cervical spine trauma where the patient's neurological status is not changing. For example, a patient with facet dislocation requiring traction and/or reduction.

- Patient requires observation in a neurosurgical ICU, but remains stable neurologically. This applies to diagnoses such as a basal ganglia hemorrhage.

- Patient does not have an established diagnosis due to lack of MRI, angiography, etc. at the referring institution but remains neurologically stable.

\section{PRE-TRANSPORT COORDINATION}

\section{Communication is Key}

- Establish continuity of care by physician-to-physician and nurse-to-nurse review of patient condition and current treatment plan. Receiving physician should provide advice to aid in pretransport stabilization and provide advance medical treatment when appropriate (ie, Mannitol, steroids, antiepileptic in setting of aneurysmal SAH with hydrocephalus). The appropriate arrangements should be made at the receiving institution to avoid delays in definitive treatment (ie, operating room standby). The mode of transport must also be established. Communication is especially critical in the setting of the first set of diagnoses described above.

\section{TRANSPORT PERSONNEL}

\section{Education = Empowerment to Intervene}

- A minimum of two people should accompany a critically ill patient consisting of a critical care nurse plus a technician. In the case of an unstable patient, it is recom- mended that a physician with training in airway management and ACLS be present. When this is not possible, some hospitals prophylactically intubate those patients who have a high risk for becoming unstable en route. In the setting of elevated ICP, the transport personnel must understand the diagnosis and means of controlling ICP during transport. For example, most patients arrive flat and supine which is not the optimal position for a patient with impending hydrocephalus. Transport personnel cannot serve as technicians, but must be aware of the patients diagnosis in order to best maintain and even optimize the patient prior to arrival.

\section{TRANSPORT EQUIPMENT}

Continuity of Care

- The patient must be maintained using the same monitors and drugs (if necessary) as are present in the ICU. A blood pressure monitor, pulse oximeter, cardiac monitor/ defibrillator, basic rescucitation drugs, and a portable mechanical ventilator when indicated.

\section{DOCUMENTATION:}

What is the Goal of Transfer?

- The patient's medical record and relevant laboratory and radiographic studies must be copied for the accepting facility. It is also suggested to perform a COBRA/EMTALA checklist to ensure compliance with federal regulations. This includes documentation of initial medical evaluation and stabilization procedures, informed consent disclosing risks and benefits of transfer, and documentation of physician-to-physician communication with the names of each physician involved. Financially motivated transfers are illegal.
The above five points serve as a general set of guidelines that should be evaluated and refined regularly by the hospital using a standard quality improvement process. The hospital transfer center should develop a checklist for each of the five points described above and follow the same steps for each and every transfer. Attention to details by methodically following the same steps in each situation will hopefully minimize "systems" mistakes and thus not only improve efficiency, but also maximize safety. 